\title{
Velocity-Resolved Echo Mapping
}

\author{
Keith Horne \\ School of Physics and Astronomy, North Haugh, St. Andrews KY16 \\ gSS, Scotland, UK
}

\begin{abstract}
We probe the structure and kinematics of Seyfert broad-line regions by using a velocity-delay map to fit simultaneously the observed variations in the continuum and the emission-line profiles. The velocitydelay map of CIV $\lambda 1550$ emission constructed from 44 IUE spectra of NGC 4151 suggests virial motions, with $10^{7} M_{\odot}$ inside two light days. Pure inflow and outflow kinematics are ruled out by the approximate symmetry of the map. A stronger redshifted response at small delays suggests either a component with free-fall kinematics, or an outflow with C IV being emitted inward by optically thick gas clouds.
\end{abstract}

\section{Echo Mapping}

Echo mapping exploits light travel-time delays to probe the size, structure, and kinematics of emission-line regions in the unresolved active nuclei of Seyfert galaxies. The required time-resolved spectrophotometry with 1 to 4 -day sampling sustained for several months has been acquired by the International AGN Watch collaboration in a series of monitoring campaigns with $I U E$ and a network of ground-based telescopes that have focussed on one object per year since 1989.

The Seyfert nuclei vary erratically in time, with power density spectra having power-law slopes of -1 to -2 . Optical and ultraviolet continua vary synchronously to $<1$ day, implying continuum production inside 1 light day, and justifying use of the observable $I U E$ /optical continua to trace of the unobservable ionizing flux.

Emission lines track continuum variations, but with smaller fractional amplitudes and time-delayed maxima and minima, consistent with photoionization driving. At densities above $10^{8} \mathrm{~cm}^{-3}$, recombination times $<1$ day imply time delays dominated by light-travel time on the longer path from nucleus to photoionized gas to the observer versus the direct path from nucleus to observer.

By careful analysis of observed light curves and line-profile variations, we recover the velocity-delay map $\Psi(v, \tau)$, which gives the delay distribution of the emission-line response in each velocity bin. This resolves the emission-line region on isodelay surfaces, which are paraboloids wrapped around the line of sight to the nucleus, and Doppler-shift surfaces, which depend on the unknown velocity field.

A maximum entropy fit recovers the velocity-delay map $\Psi(v, \tau)$, along with the driving continuum light curve $C(t)$, bridging gaps in the data as necessary. 


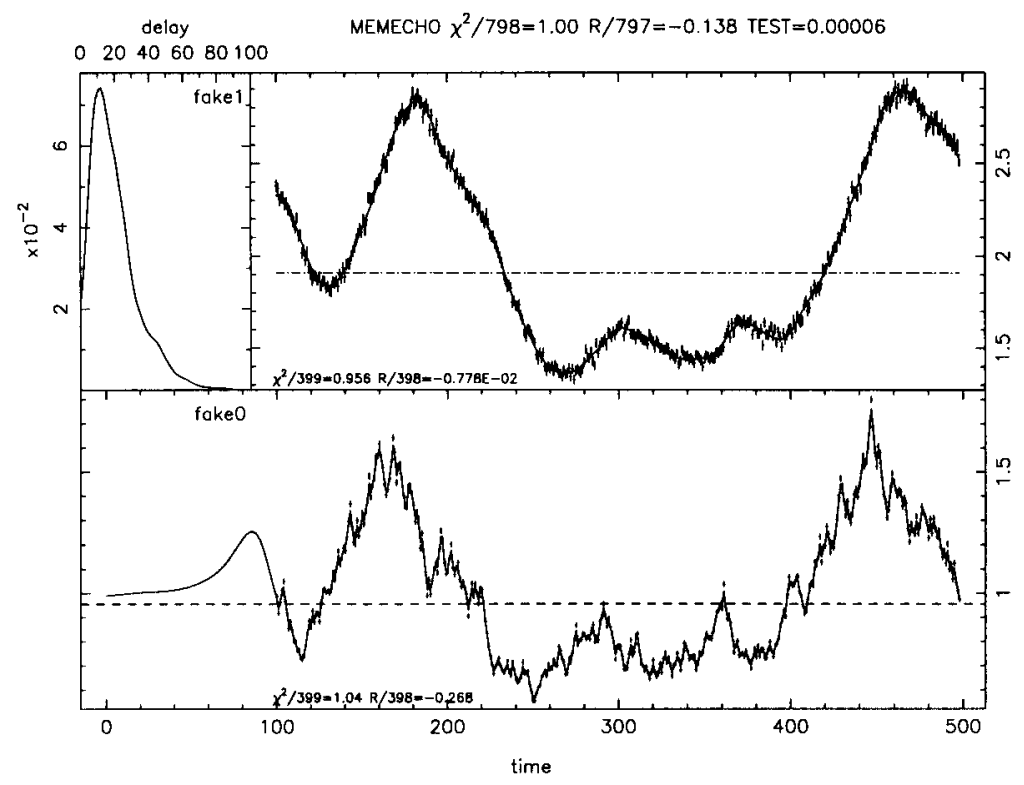

Figure 1. MEMECHO reconstruction of a 1-dimensional delay map from simulated light curves. The reconstructed map closely resembles the true map $\Psi(\tau) \propto \tau e^{-\tau}$.

The line-profile variations $L(v, t)$ are given by

$$
L(v, \tau)=\bar{L}(v)+\int_{\tau_{\min }}^{\tau_{\max }} \Psi(v, \tau)[C(t-\tau)-\bar{C}] d \tau,
$$

were $\bar{L}(v)$ and $\bar{C}$ are time-steady line and continuum backgrounds. The $\chi^{2}$ statistic judges the fit. With $\bar{C}$ fixed at an arbitrary value, $\bar{L}(v), C(t)$, and $\Psi(\tau)$ are adjusted until $\chi^{2} / N=1$, where $N$ is the number of data points. The fit is not unique, but the maximum-entropy criterion 'steers' each point on $\bar{L}(v), C(t)$, and $\Psi(v, \tau)$ toward its neighbors, delivering the 'smoothest' positive functions that fit the data. This method implemented in the computer code MEMECHO is discussed in detail elsewhere (Horne 1994).

In Fig. 1, MEMECHO fits a delay map to simulated continuum and line light curves, recovering the true map $\Psi(\tau) \propto \tau e^{-\tau}$ in some detail. MEMECHO has been used to make delay maps of optical and ultraviolet emission lines in NGC 5548 (Horne, Welsh, \& Peterson 1991, Krolik et al. 1991, Wanders et al. 1995), Mrk 590 (Peterson et al. 1993), NGC 3516 (Wanders \& Horne 1994), and NGC 4151 (Ulrich \& Horne 1996).

\section{Velocity-Delay Maps}

Figure 2 sketches the formation of the velocity-delay map for spherical free-fall into a point-mass potential, and for a Keplerian disk (Welsh \& Horne 1991, Perez, Robinson, \& de la Fuente 1992). The dramatically different appearance 


\section{Freefall Disk}

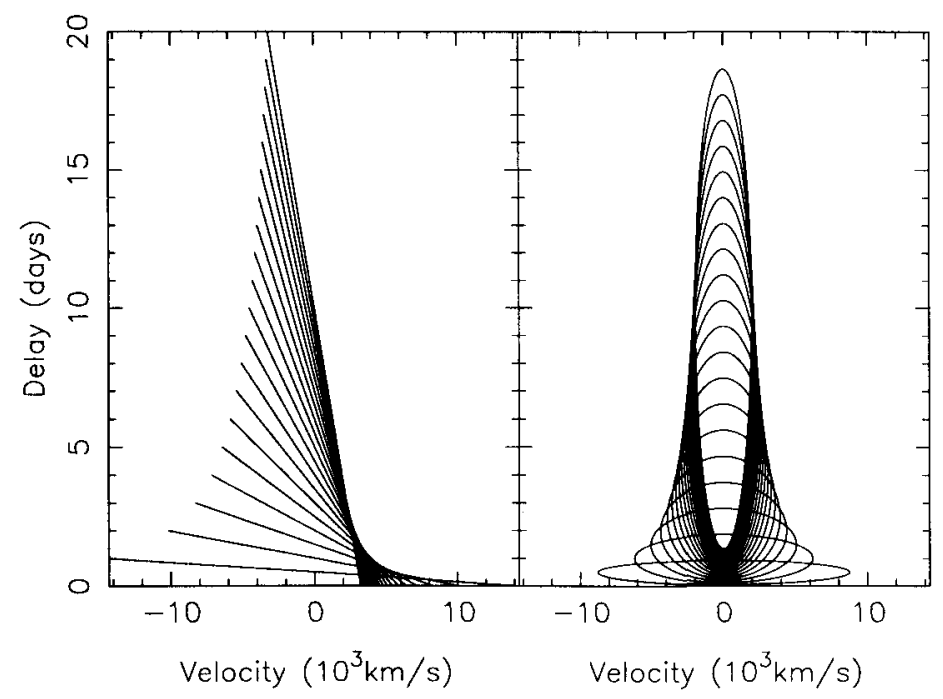

Figure 2. Velocity-delay maps for spherical free-fall and Kepleriandisk kinematics. Spherical shells map to diagonal lines, and disk annuli map to ellipses. The central mass is $10^{7} M_{\odot}$ in both cases. The disk inclination angle is $60^{\circ}$.

of the two flows highlights the power of velocity-delay maps for kinematic diagnosis.

Any spherically symmetric flow is a nested set of thin spherical shells. The time delay $\tau$ and Doppler shift $v$ are

$$
\tau=(1+\cos \theta) 2 R / c, \quad v=v_{R} \cos \theta,
$$

with $R$ the shell radius, $\theta$ the angle from the back edge, and $v_{R}$ the outflow velocity. The linear dependence of both $\tau$ and $v$ on $\cos \theta$ implies that each shell maps into a diagonal line in the velocity-delay plane, as shown in Fig. 2.

The circulating disk flow is a set of concentric co-planar cylindrical annuli. The time delay and Doppler shift are

$$
\tau=(1+\sin i \sin \phi) 2 R / c, \quad v=v_{\phi} \sin i \cos \phi,
$$

with $R$ and $i$ the radius and inclination of the annulus, $\phi$ the azimuth, and $v_{\phi}$ the azimuthal velocity. Each annulus maps to an ellipse on the velocity-delay plane, as in Fig. 2. Inner annuli map to 'squashed' ellipses (large $\pm v$, small $\tau$ ), while outer annuli map to 'stretched' ellipses (small $\pm v$, large $\tau$ ).

Even though the velocity-delay map projects a 6-dimensional phase space onto a 2-dimensional plane, it can still provide a good impression of flow when the velocity field is ordered and has some degree of symmetry. The envelope of the velocity-delay map can reveal the presence of virial motions $v^{2} \propto \sqrt{G M / c \tau}$, with $M$ the mass of the central object. A red/blue asymmetry or lack thereof 


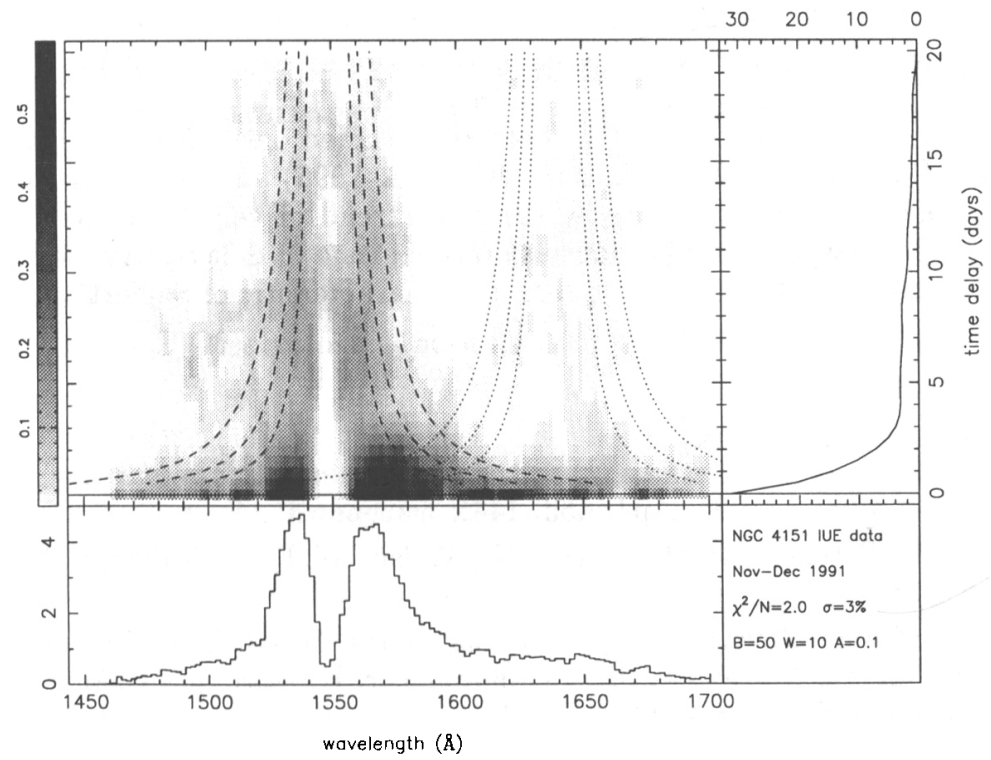

Figure 3. MEMECHO velocity-delay map of C IV $\lambda 1550$ emission (and superimposed He II $\lambda 1640$ emission) in NGC 4151. Dashed curves give escape velocity for masses $0.5,1.0$, and $2.0 \times 10^{7} M_{\odot}$.

gauges the importance or absence of radial flow relative to azimuthal motions. A disordered velocity field with a range of velocities at each position smears the map in the $v$ direction. The far side of the map (large $\tau$ ) is enhanced by anisotropic emission from optically thick clouds that radiate lines inward toward the nucleus (Ferland et al. 1992, O'Brien, Goad, \& Gondhalekar 1994).

\section{Velocity-Delay Map of C IV Emission in NGC 4151}

Figure 3 shows the velocity-delay map for C IV $\lambda 1550$ and He II $\lambda 1640$ emission based on 44 IUE spectra of NGC 4151 covering 22 epochs during 1991 Nov 9Dec 15 (Ulrich \& Horne 1996). Although spanning only 36 days, this campaign recorded favorable continuum variations, including a bumpy exponential decline followed by a rapid rise, which were sufficient to support echo mapping on delays from 0 to 20 days.

Disregard the strong C IV absorption feature, which obliterates the delay structure at small velocities. The wide range of velocities at small delays and smaller range at larger delays suggests virial motions. The dashed curves in Fig. 3 give escape velocity envelopes $v=\sqrt{2 G M / c \tau}$ for masses $0.5,1.0$, and $2.0 \times 10^{7} M_{\odot}$. A mass of order $10^{7} M_{\odot}$ may be concentrated within 1 light day of the nucleus.

The approximate red-blue symmetry of the CIV map rules out pure inflow or outflow kinematics. The somewhat stronger $\mathrm{C}$ IV response on the red side at small delays and on the blue side at larger delays suggests a gas component with free-fall kinematics. However, if the C IV emission arises from the irradiated faces 
of optically thick clouds, those on the far side of the nucleus will be brighter, and the redward asymmetry can then be interpreted as an outflow combined with the inward C IV anisotropy.

Velocity-delay maps have so far been constructed only for CIV emission in NGC 5548 (Wanders et al. 1996, Done \& Krolik 1996) and NGC 4151 (Ulrich \& Horne 1996). Both systems show the same trend of velocity dispersion decreasing with delay, and red response stronger at small delays. As more maps are constructed we will see if this is a general characteristic of Seyfert broad-line regions.

\section{References}

Done, C., \& Krolik, J. 1996, ApJ, 463, 144.

Ferland, G.J., Peterson, B. M., Horne, K., Welsh, W.F., \& Nahar, S. N. 1992, ApJ, 387, 95.

Horne, K. 1994, in Reverberation Mapping of the Broad-Line Region in Active Galactic Nuclei, ed. P. M. Gondhalekar, K. Horne, \& B. M. Peterson (ASP: San Francisco), 23.

Horne, K., Welsh, W.F., \& Peterson, B. M. 1991, ApJL, 367, L5.

O'Brien, P.T., Goad, M.R., \& Gondhalekar, P. M. 1994, MNRAS, 268, 845.

Peterson, B. M., Ali, B., Horne, K., Bertram, R., Lame, N.J., Pogge, R.W., \& Wagner, R. M. 1993, ApJ, 402, 469.

Pérez, E., Robinson, A., \& de la Fuente, L. 1992, MNRAS, 256, 103.

Krolik, J. H., Horne, K., Kallman, T. R., Malkan, M. A., Edelson, R. A., \& Kriss, G. A. 1991, ApJ, 371, 541.

Ulrich, M.-H., \& Horne, K. 1996, MNRAS, in press.

Wanders, I., \& Horne, K. 1994, A\&A, 289, 76.

Wanders, I., Goad, M.R., Korista, K.T., Peterson, B.M., Horne, K., Ferland, G. J, Koratkar, A.P., Pogge, R.W., \& Shields, J.C. 1995, ApJL, 453, L87.

Welsh, W. F., \& Horne, K. 1991, ApJ, 379, 586. 